\title{
Unconscious Biometrics for Continuous User Verification
}

\author{
Isao Nakanishi \\ Graduate School of Engineering, \\ Tottori University \\ 4-101 Koyama-minami, Tottori-shi \\ +81-857-31-5132 \\ nakanishi@eecs.tottori-u.ac.jp
}

\begin{abstract}
In user management system, continuous or successive (ondemand) authentication is required to prevent identity theft. In particular, biometrics of which data are unconsciously presented to authentication systems is necessary. In this paper, brain waves and intra-palm propagation signals are introduced as biometrics and their verification performances using actually measured data are presented.
\end{abstract}

\section{CCS Concepts}

- Security and privacy Biometrics

\section{Keywords}

Unconscious Biometrics; Continuous Authentication; Successive Authentication; Brain Wave, Intra-palm Propagation Signal;

\section{INTRODUCTION}

Biometrics using the fingerprint, iris, and vein are not suitable for applications to user management of systems since they are based on one-time-only authentication while they achieve higher authentication performance. After authenticating by a genuine user of a system and removing any limitations for using the system, it is easy for others to use the system instead of the genuine user.

In order to prevent such identity theft, continuous or successive (on-demand) authentication is needed $[1,2]$. However, biometrics of which data are unconsciously (transparently) presented to authentication systems is required to perform the continuous or successive authentication. In continuous authentication, we must keep presenting biometric data to a sensor for authentication while using a system. In successive authentication, we must present biometric data to a sensor on demand of authentication. These degrade the usability of systems. Because of the similar reasons, conventional authentication methods: the password and the ID card are unable to perform continuous or successive authentication. Continuous authentication is realized by only using biometrics. In other words, biometrics is best suitable for continuous authentication.

Permission to make digital or hard copies of all or part of this work for personal or classroom use is granted without fee provided that copies are not made or distributed for profit or commercial advantage and that copies bear this notice and the full citation on the first page. Copyrights for components of this work owned by others than ACM must be honored. Abstracting with credit is permitted. To copy otherwise, or republish, to post on servers or to redistribute to lists, requires prior specific permission and/or a fee. Request permissions from Permissions@acm.org.

ICSPS '16, November 21-24, 2016, Auckland, New Zealand (C) 2016 ACM. ISBN 978-1-4503-4790-7/16/11 .\$15.00 DOI: http://dx.doi.org/10.1145/3015166.3015180
As unconsciously presentable biometrics, there are two types. One is biometrics of which data can be measured not actively but passively. Biometric data of the face or the ear can be detected by using a sensor (camera) even when users are not aware of presenting the data. In other words, it suggests that it is easy to detect the biometric data by others while users are unaware. Fake faces or ears are made by using such secretly-captured data and then used in identity theft. The other is biometrics which is detectable from users' continuous action. For example, the voice print in speaking, the gait in walking, the key-stroke in typing, and so on. However, their applications are limited in those actions.

In this paper, we focus attention on brain waves which are not surfaced on the body and detectable passively. The brain wave is electrical potential (EEG) on a scalp which is generated by brain activities and always measurable unconsciously. On the other hand, users are generally controlling systems by touching or gripping a part of the systems. Thus, we assume touching or gripping as more common user's action and propose to use intrapalm propagation signals as biometrics.

\section{USER VERIFICATION UISNG EEG}

The brain wave is unconsciously generated; therefore, it is a candidate of transparent biometrics which is applicable to continuous authentication. However, when users set brain wave sensors on their scalp, they are aware of wearing the sensors. It is impossible to detect brain waves by using contactless sensors. However, once they set the sensors, it is possible to unconsciously measure brain waves and to measure brain waves does not prevent users from using systems. In addition, the brain wave is biometrics which has the highest acceptability since any physically-disabled people certainly have brain waves.

To use the brain wave as biometrics have been studied [3-14]. However, almost all of them are still in the basic research stage and do not assume concrete applications. It is necessary to wear sensors whenever detecting brain waves; therefore, the brain wave as biometrics is not suitable for applications based on one-timeonly authentication, where users are authenticated once when they enter a room, use a computer, and so on. This issue is not fully discussed in conventional studies. In some cases, dozens of sensors are used for achieving higher authentication performance. It is not assumable in practical applications. It also increases computational mount for authentication. Moreover, the use of response to visual stimuli in brain waves is researched in Ref. $[12,13]$. To give visual stimuli to users makes them be aware of presenting biometric data; therefore, it is unsuitable for continuous or successive authentication.

\subsection{Toward Driver Authentication}

Brain waves as biometrics are suitable for continuous or successive user authentication of a system, especially a higher secured system. For example, transportation system as trains, 


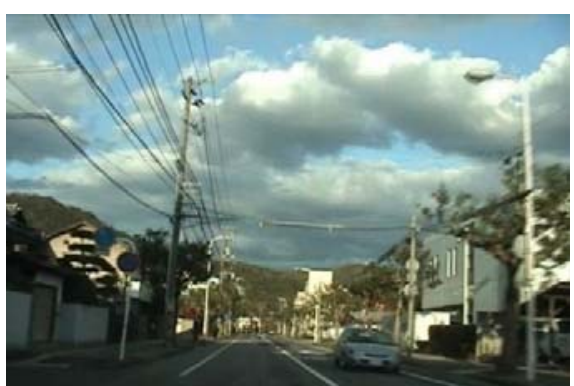

Figure 1. A capture scene in the route tracing.

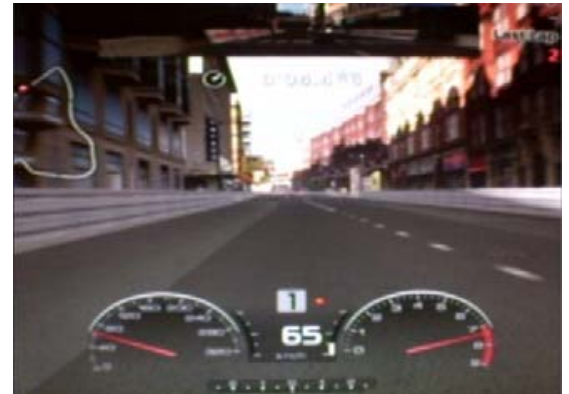

Figure 2. A captured image in the simplified driving simulator.

buses, and airplanes which involve many human lives, computer systems with sensitive information, remote education systems for qualification or license, and so on. In this subsection, assuming driver authentication, we introduce the measurement of brain waves in two simulated driving environments and results of verification performance $[15,16]$.

\subsubsection{Route Tracing}

When drivers move to their known places, they select a path by checking actual scenes against their memorized ones. Such a mental process is extracted and regarded as a mental task called route tracing [15]. This task involves no body movement and not to imagine driving. In order to obtain actual driving scenes, a video was captured by using a digital video camera which was settled on a dashboard in a car. A driving scene in the captured video is shown in Fig. 1. The video was displayed on a wide screen by using a projector.

\subsubsection{Simplified Driving Simulator}

In order to create a similar environment with actual driving one in my laboratory, we used handle, accelerator and brake controllers of a gaming machine. The driving scenes were generated by a racing game software and displayed to the wide screen by using the projector. A racing mode was "urban road" assuming to drive on general roads. The speed limit was set to 65 $\mathrm{km}$. "time-trial mode" (one car racing) was selected to escape accidents caused by other cars. A captured driving scene is shown in Fig. 2.

\subsubsection{Brain Wave Sensor}

In these experiments, we used a consumer headband type brain wave sensor. The number of sensors was one and its measuring point was the frontal region of head which is defined as $\mathrm{fp} 1$ in the international standard: ten-twenty electrode system. It is easy and practicable to set a sensor on the frontal region of head

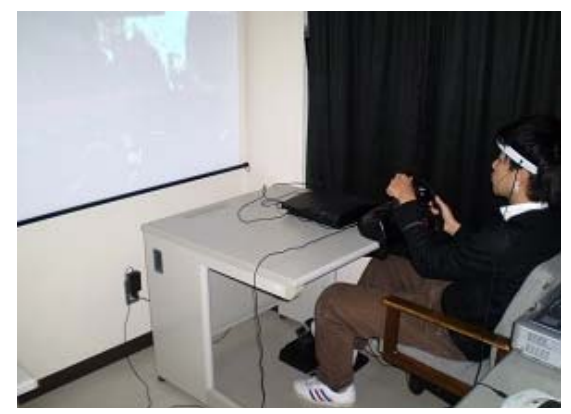

Figure 3. A measurement scene in the simplified driving simulator.

since setting the sensor is not disturbed by user's hair. The specification of the sensor is shown in Table 1 .

Table 1. Specification of the brain wave sensor

\begin{tabular}{|l|l|}
\hline & Brain Builder Unit \\
\hline Frequency Range & $1-24 \mathrm{~Hz}$ \\
\hline Voltage Range & $5-80 \mu \mathrm{Vpp}$ \\
\hline Sampling Frequency & 128 \\
\hline
\end{tabular}

\subsubsection{Measurement of Brain Waves}

The number of experimental subjects was thirty. All were students of my university around twenty years old and had a driving license. In the route tracing, they were presented with the captured driving video and required to memorize the discriminative points: directions, intersections, and landmarks. After that, they wore the brain wave sensor and then were presented with the same driving video. They were requested to simulate their driving and in the meantime their brain waves were measured. In the simplified driving simulator, the subjects practiced the simulator in two laps to get used to it before measurement. When accidents were occurred, the measurement was canceled and restarted from the beginning. The measurement scene was shown in Fig. 3. In both environments, the size of screen was 60 inch. The distance between subjects and the screen was $130 \mathrm{~cm}$. The measurement time was three minutes. Measuring was achieved twice a day and it was repeated five times. As a result, there were ten data from each subject in each environment.

\subsection{Feature Extraction and Verification}

Figure 4 shows a procedure of verification. In the enrollment stage, brain waves are measured from all registered users and then their spectra are obtained by using FFT. Transformation coefficients for each principle component are obtained by applying Principle Component Analysis (PCA) to the spectra. Using the transformation coefficients, a principal component score for each spectrum is calculated. Transformation coefficients for the selected principle components are memorized in the system. At the same time, using the cumulative contribution ratio, the number of principle components is determined. Principle component scores in the selected principle components are used as individual features. Verification is achieved by Support Vector Machine (SVM) [17], especially one versus one SVM (1vs1 SVM) [18]. SVM is a powerful two-class classifier based on learning. From all users, a pair (two users) is selected, and one is 


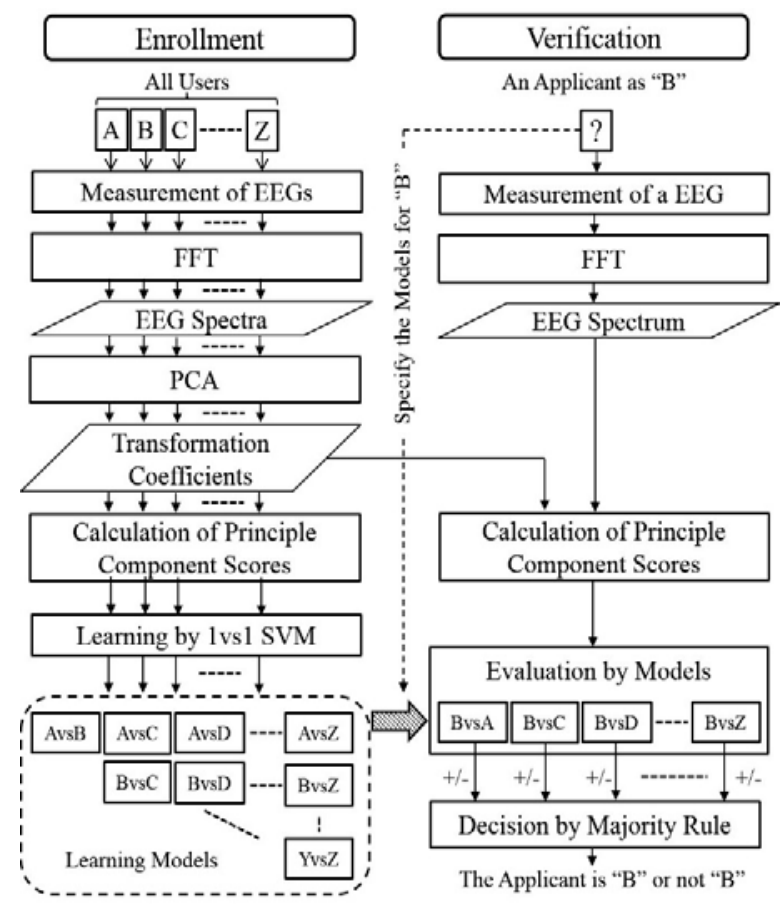

Figure 4. Block diagram of verification using brain waves.

assumed to be a registered user and another is assumed to be a nonregistered user. By giving ' +1 ' to a principle component score of the registered user and ' -1 ' to the score of the nonregistered user, a SVM model for classifying the two users is learned. In this way, SVM models for all possible pairs are constructed and memorized in the system. In the verification stage, an applicant specifies a registered user by indicating his /her name to the system. A brain wave is measured from the applicant and its spectrum is calculated. Using the spectrum and the transformation coefficients memorized in the system, principle component scores are calculated and then evaluated in SVM models which are related with the registered user specified by the applicant. Outputs of the SVM models are real number and so coded to ' +1 ' or ' -1 ' by using the sign function. When the number of models of which output are ' +1 ' is larger than a threshold, the applicant is regarded as a registered user.

\subsection{Performance Evaluation}

Using brain waves measured from thirty subjects in two simulated driving environments, verification performance was evaluated using Equal Error Rate (EER), where False Acceptance Rate (FAR) is equal to False Rejection Rate (FRR). The cumulative contribution ratio in PCA was set to $70 \%$ through preliminary examinations. The number of learning data was seven from each subject while the number of test data was three. Optimal kernel functions and parameters for constructing SVM models were found through the grid searching. As a result, the EER in the route tracing was $22 \%$ and the EER in the simplified driving simulator was $24 \%$ [19].

\section{USER VERIFICATION UISNG INTRA- PALM PROPAGATION SIGNALS}

The intra-palm propagation signal is another transparent biometrics. An electrode pair is set on a palm and then an

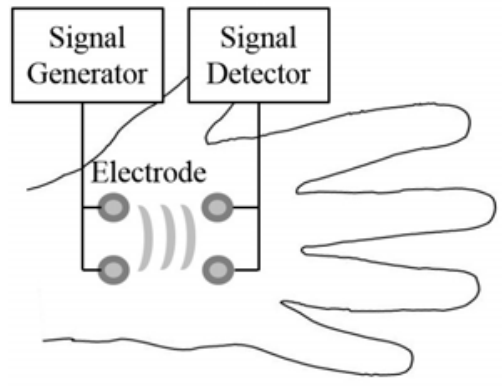

Figure 5. Intra-palm propagation signal.

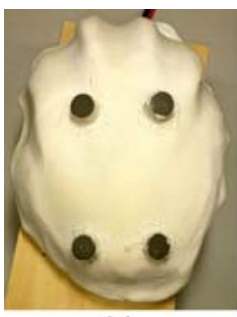

(a)

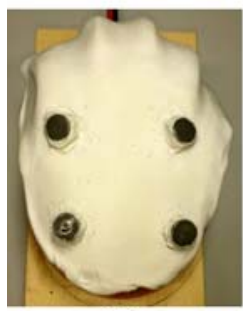

(d)

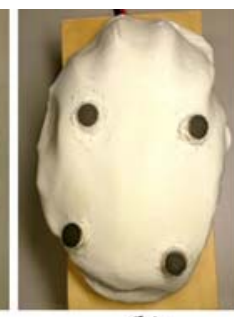

(b)

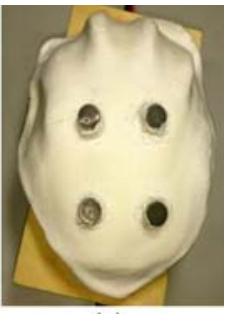

(c)

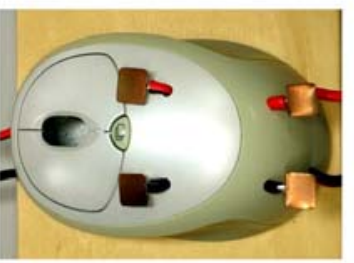

(e)

Figure 6. Measuring devices for intra-palm propagation signals.

alternating electric current is passed through the electrodes. This yields electric field variation around the electrodes and it is detected as voltage variation by another electrode pair. This mechanism is diverted from intra-body communication technology. The voltage variation is regarded as a propagated signal between the electrode pairs and called intra-palm propagation signal in convenience. The fundamentals are described in Fig. 5. If the propagation characteristic of each user is different from those of others, the intra-palm propagation signal can be used as biometrics.

\subsection{Measuring Devices}

Devices for measuring intra-palm propagation signals were made as shown in Fig. 6. Handshapes while naturally grasping something were molded out of clay and their plaster casts were made as bases. When users lay their hands on each base, electrodes on the base are naturally contacted to their palms. Commercially produced disposal electrodes $(\mathrm{Ag} / \mathrm{AgCl})$ with removing gelled pads were used in these devices. There were four different arrangements in the electrodes: (a) $2 \mathrm{~cm} \times 5 \mathrm{~cm}$, (b) 4 $\mathrm{cm} \times 5 \mathrm{~cm}$, (c) $2 \mathrm{~cm} \times 3 \mathrm{~cm}$, (d) $4 \mathrm{~cm} \times 3 \mathrm{~cm}$. The device (e) was based on a mouse device and each electrode was made from a cupper plate. The arrangement of electrodes was $2 \mathrm{~cm} \times 5 \mathrm{~cm}$. 


\subsection{Measuring Equipment}

A source signal was a quasi-white Gaussian signal with the range of $100 \mathrm{MHz}$. As an individual feature, power spectra of propagated signals were used in verification. The reason why to use a white Gaussian signal is to efficiently get propagation characteristics (spectra). Specifications of measuring equipment is shown in Table 2.

Table 2. Specifications of measuring equipment

\begin{tabular}{|c|c|}
\hline $\begin{array}{c}\text { Signal } \\
\text { Generator }\end{array}$ & $\begin{array}{c}\text { Signal Oscillator } \\
\text { Quasi-White Gaussian Signal } \\
\text { Frequency Range: } 100 \mathrm{MHz}\end{array}$ \\
\hline \multirow{3}{*}{ Signal } & Digital Oscilloscope \\
Fetector & Sampling Rate: $1 \mathrm{GSa} / \mathrm{s}$ \\
& Memory Size: $32 \mathrm{Mpts}$ \\
\hline
\end{tabular}

\subsection{Feature Extraction}

Power spectra of measured propagation signals were used as individual features as mentioned above. However, propagation spectra have variation even in an identical user. This causes degradation of verification performance. Thus, averaging and normalizing were applied to the spectra.

Averaging is performed as follows. A measured signal is equally divided into several regions. In each region, a mean value is calculated and then a DC component is removed by subtracting the mean value form each amplitude. The DC component may be very larger than $\mathrm{AC}$ components; therefore, it becomes disadvantageous in normalization. An amplitude spectrum is calculated by FFT in each region and then obtained spectra in all regions are ensemble-averaged. As a result, an averaged propagation spectrum is obtained. Normalizing is achieved by equalizing mean values of all propagation spectra. The reason is not to absolutely compare amplitude spectra but to relatively compare them.

The number of spectral values in a propagation spectrum at 0 $-100 \mathrm{MHz}$ is one hundred. The frequency band is divided into ten parts $(0-10 \mathrm{MHz}, 10-20 \mathrm{MHz}, \ldots, 90-100 \mathrm{MHz})$ and any one of them, that is, ten spectral values which is optimal for each user is used as individual features for verification. Which part is optimum for each user is empirically investigated in advance of verification.

\subsection{Verification}

As used in the case of user verification using EEG, 1vs1 SVM is also used but PCA is not used in this case. The flowchart of verification process is shown in Fig. 7. In the enrollment stage, Each SVM model is learned using ten spectral values in an optimal part (band) for each user. In the verification stage, ten spectral values in the same optimal part are evaluated in SVM models which are related with a user specified by an applicant. Final decision is done based on a majority rule using outputs of the SVM models.

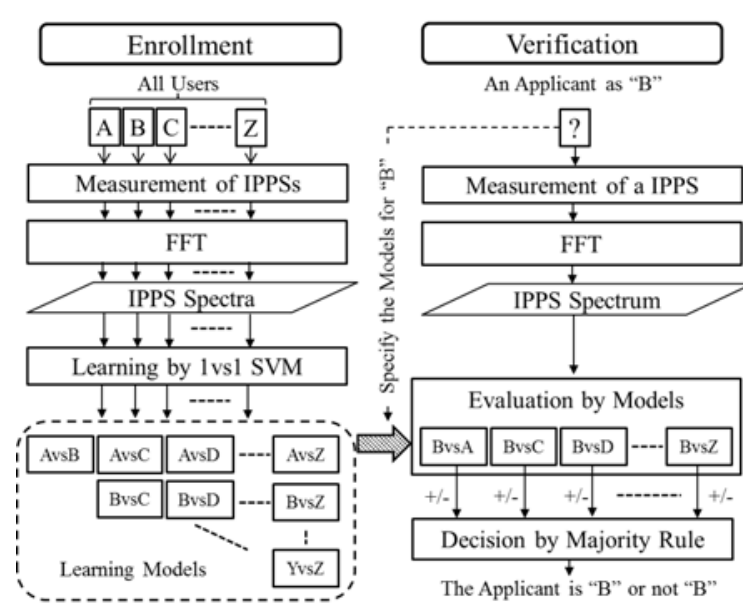

Figure 7. Measuring devices for intra-palm propagation signals (IPPSs).

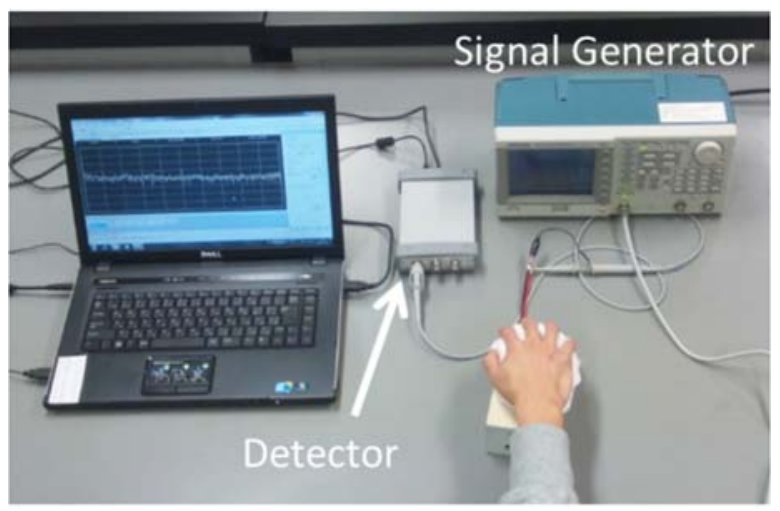

Figure 8. A measuring scene of intra-palm propagation signals.

\subsection{Performance Evaluation}

\subsubsection{Measurement of Propagation Signals}

Experimental subjects were male students in my university and its number was twenty-one. They sat a chair and relaxed, and then wiped their right-hand palms using an alcohol-soaked tissue paper. After that, they set their right-hand palm on measuring devices checking position of electrodes on the palms and then their propagation signals were measured. The measurements were done twice a day keeping interval of five hours and that was repeated thirty times (days). In total, sixty propagation signals were obtained from each subject.

A measuring scene is shown in Fig. 8. The laptop computer controls a signal detector as a digital oscilloscope and saves measured propagation signals as digital data. An example of propagation spectrum is shown in Fig. 9. 


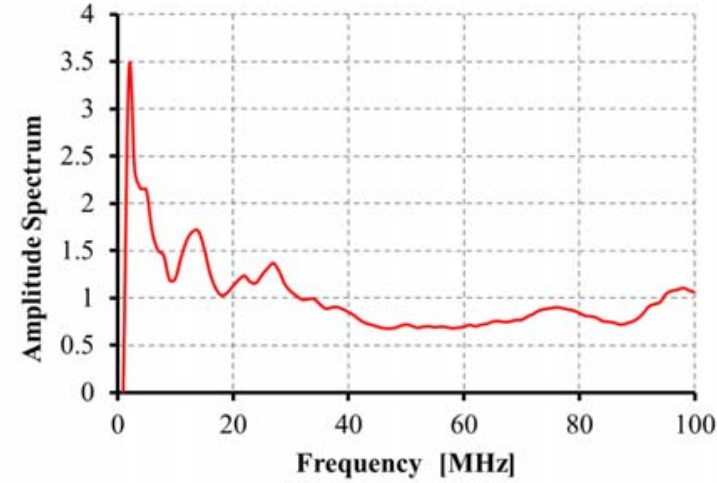

Figure 9. An example of intra-palm propagation spectrum.

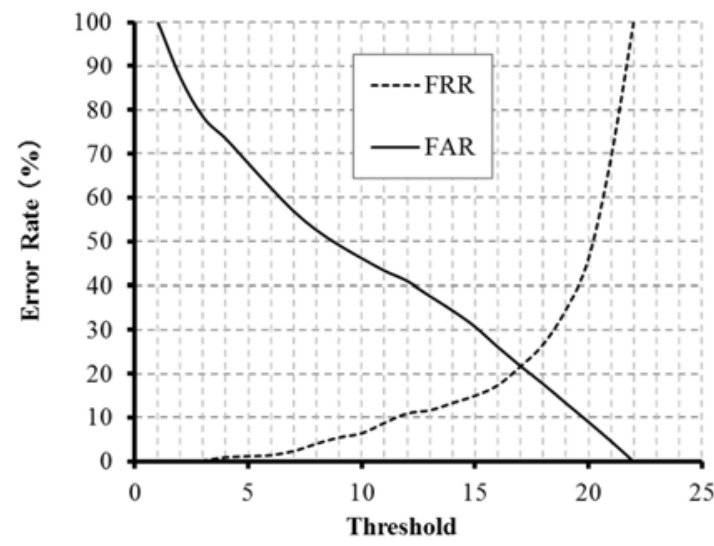

Figure 10. Error rate curves in a measuring device.

\subsubsection{Verification Performance:}

The number of learning data in SVM was 40 for genuine users and 40 for the others. Optimal parameters for constructing each SVM model were found in the grid searching from the values shown in Table 3. The number of test data in SVM was twenty for genuine users and four hundred $(20 \times 20)$ for the others.

As a result, a mean value in EERs in five measuring devices was $24 \%$ and the variation was small. It is confirmed that intrapalm propagation signals can be used as a biometrics. However, the EER was not satisfactory. Error rate curves in a measuring device is shown in Fig. 10. It is clear that FAR is high. In order to improve verification performance, FAR must be degreased. To reduce intra-individual variation is a future problem.

Table 3. Parameter values in grid searching.

\begin{tabular}{|l|l|l|}
\hline \multicolumn{2}{|l|}{$C$ Parameter } & $0.001,0.002, \cdots, 0.009$, \\
& $0.010,0.015, \cdots, 0.095$, \\
& $0.10,0.15, \cdots, 0.95$, \\
& $1.0,2.0, \cdots, 9.0,10.0$ \\
\hline & Polynomial:d & $1,2,3$ \\
\cline { 2 - 3 } Kernel & & $0.001,0.002, \cdots, 0.009$, \\
Function & \multirow{2}{*}{ RBF $: \delta$} & $0.010,0.015, \cdots, 0.095$, \\
& & $0.10,0.15, \cdots, 0.95$, \\
& & $1.0,2.0, \cdots, 9.0,10.0$ \\
\hline Frequency Band (MHz) & $1-10,11-20, \cdots, 91-100$ \\
\hline
\end{tabular}

\section{CONCLUSIONS}

The use of brain waves as biometrics was introduced. The brain wave is not surfaced on the body and passively-detectable; therefore, it has a tolerability to identity theft using artifacts which are made by using secretly-captured data. The verification performance in two simulated driving environments was shown. In addition, focusing on 'grasping or touching' in equipment applications as more general continuous action, intra-palm propagation signals as biometrics is introduced. Their verification performance was also shown.

However, in both approaches the verification performances are unsatisfactory. It is an urgent issue to improve the verification performances. It is also necessary to construct a continuous authentication system using them and then to evaluate their usability and acceptability.

\section{REFERENCES}

[1] Altinok A. and Turk M. 2003. Temporal Integration for Continuous Multimodal Biometrics. In Proceedings of Workshop on Multimodal User Authentication (2003), 207-214.

[2] Nakanishi I. and Miyamoto C. 2010. On-Demand Biometric Authentication of Computer Users Using Brain Waves. In Networked Digital Technologies. F. Zavoral et al. Eds. Communications in Computer and Information Science (CCIS) series of Springer LNCS, 87 (Jul. 2010), 504-514.

[3] Poulos M., Rangoussi M., Chrissikopoulos V., and Evangelou A. 1999. Person Identification Based on Parametric Processing of the EEG. In Proceedings. of the 9th IEEE International Conference on Electronics, Circuits and Systems, 1 (1999), 283-286.

[4] Poulos M., Rangoussi M., and Alexandris N. 1999. Neural Networks Based Person Identification Using EEG Features. In Proceedings of International Conference on Acoustic Speech and Signal Processing, (1999), 1117-1120.

[5] Poulos M., Rangoussi M., Chissikopoulus V., and Evangelou A. 1999. Parametric Person Identification from the EEG Using Computational Geometry. In Proceedings of the 6th IEEE International Conference on Electronics, Circuits and Systems, (1999), 1005-1008.

[6] Joachims T. 2008. SVM-light Support Vector Machine. http://svmlight.joachims.org.

[7] Nakanishi I. and Yoshikawa T. 2015. Brain Waves as Unconscious Biometrics towards Continuous Authentication - The Effects of Introducing PCA into Feature Extraction - In Proceedings of IEEE International Symposium on Intelligent Signal Processing and Communication Systems, (Nov. 2015), 422-425.

[8] Paranjape R. B., Mahovsky J., Benedicent L., and Koles Z. 2001. The Electroencephalogram as a Biometric. In Proceedings of Canadian Conference on Electrical and Computer Engineering, 2 (2001), 1363-1366.

[9] Ravi K. V. R. and Palaniappan R. 2005. Recognition Individuals Using Their Brain Patterns In Proceedings of the 3rd International Conference on Information Technology and Applications, (2005). 
[10] Palaniappan R. Identifying Individuality Using Mental Task Based Brain Computer Interface. In Proceedings of the 3rd International Conference on Intelligent Sensing and Information Processing, (2005), 239-242.

[11] Palaniappan R. Multiple Mental Thought Parametric Classification: A New Approach for Individual Identification. International Journal of Signal Processing, 2, 1 (2005), 222-225.

[12] Mohammadi G., Shoushtari P., Ardekani B. M., and Shamsollahi M. B. Person Identification by Using AR Model for EEG Signals. In Proceedings of World Academy of Science, Engineering and Technology, 11, 2 (2006), 281-285.

[13] Marcel S. and Millan J. R. 2007. Pearson Authentication Using Brainwaves (EEG) and Maximum A Posteriori Model Adaption. IEEE Trans. on Pattern Analysis and Machine Intelligence, 29, 4 (2007), 743-748.

[14] Palaniappan R. and Mandic D. P. 2007. Biometrics from Brain Electrical Activity: A Machine Learning Approach. IEEE Trans. on Pattern Analysis and Machine Intelligence. 29, 4 (2007), 738-742.

[15] Singhal G. K. and Ramkumar P. 2007. Person Identification Using Evoked Potentials and Peak Matching. In Proceedings of Biometric Symposium (2007).

[16] Riera A., Soria-Frish A., Caparrini M., Grau C., and Ruffini G. Unobtrusive Biometrics Based on Electroencephalogram Analysis. EURASHIP Journal on Advances in Signal Processing, 2008, 143728 (2008), 1-8.

[17] Miyamoto C., Baba S., and Nakanishi I. Biometric Person Authentication Using New Spectral Features of Electroencephalogram (EEG). In Proceedings of 2008 IEEE International Symposium on Intelligent Signal Processing and Communication Systems, (2008) 312-315.

[18] Nakanishi I., Miyamoto C., and Baba S. EEG Based Biometric Authentication Using New Spectral Features. In Proceedings of 2009 IEEE International Symposium on Intelligent Signal Processing and Communication Systems, (2009), 651-654.
[19] Nakanishi I., Baba S., and Li S. Driver Authentication Using Brain Waves While Route Tracing as a Mental Task. In Proceedings of the 6th International Conference on Security and Cryptography, (2011), 90-96.

[20] Nakanishi I., Ozaki K., and Li S. Evaluation of the Brain Wave as Biometrics in a Simulated Driving Environment. In Proceedings of the International Conference of the Biometrics Special Interest Group, (2012), 351-361.

[21] Nakanishi I., Baba S., Miyamoto C. and Li S. Person Authentication Using a New Feature Vector of the Brain Wave. Journal of Communication and Computer, 9 (2012), 101-105.

[22] Nakanishi I., Miyamoto C. and Li S. Brain waves as biometrics in relaxed and mentally tasked conditions with eyes closed. International Journal of Biometrics, 4, 4 (2012), 357-372.

[23] Nakanishi I., Fukuda H., and Li S. Biometric Verification Using Brain Waves toward On-Demand User Management Systems Performance differences between divided regions in alpha-beta wave band. In Proceedings of the 6th International Conference on Security of Information and Networks, (2013), 131-135.

[24] Nakanishi I. and Yoshikawa T. Brain Waves as Unconscious Biometrics towards Continuous Authentication - The Effects of Introducing PCA into Feature Extraction -. In Proceedings of IEEE International Symposium on Intelligent Signal Processing and Communication Systems, (2015), 422-425.

[25] Nakanishi I., Inada T., Sodani Y., and Li S. Performance Evaluation of Intra-Palm Propagation Signals as Biometrics. In Proceedings of International Conference on Biometrics and Kansei Engineering, (2013), 91-94.

[26] Nakanishi I., Inada T., and Li S. New Dedicated Measuring Devices for Intra-Palm Propagation Signals. In Proceedings of the International Symposium on Biometric and Security Technologies, (2014), 35-38. 


\section{Authors' background}

\begin{tabular}{|l|l|l|l|}
\hline Your Name & Title $^{*}$ & Research Field & Personal website \\
\hline Isao & full professor & $\begin{array}{l}\text { Digital Signal } \\
\text { Processing, } \\
\text { Biometrics, } \\
\text { Speech } \\
\text { Processing }\end{array}$ & $\begin{array}{l}\text { http://labs.eecs.tottori- } \\
\text { u.ac.jp/ele_sys/nakanishi/Nakanishi_e.html }\end{array}$ \\
\hline
\end{tabular}

${ }^{*}$ This form helps us to understand your paper better, the form itself will not be published.

*Title can be chosen from: master student, Phd candidate, assistant professor, lecture, senior lecture, associate professor, full professor 